\title{
Exploring extratropical transition with hybrid idealised models
}

\author{
$\underline{\text { Claire Krause }}^{\text {a }}$, Craig Arthur ${ }^{a}$ and Cindy Bruyere ${ }^{b}$ \\ ${ }^{a}$ Geoscience Australia, Canberra, Australian Capital Territory \\ ${ }^{b}$ National Center for Atmospheric Research, Boulder, United States of America \\ Email: claire.krause@ga.gov.au
}

\begin{abstract}
Tropical cyclones present a tangible risk to Australia's tropical coastal communities, however extratropical transition (ETT) of these storms can result in significant impacts in mid-latitude regions as well. Tropical systems are driven by latent heat release in the inner core of the cyclone. A fully tropical system is highly axisymmetric; with a warm-cored vortex that is readily represented by a simple radial profile (wind speed is a function of distance from the centre in all directions). Extratropical cyclones on the other hand are driven by strong thermal gradients and as a result have a highly asymmetric wind field that cannot be as easily parameterised for use in stochastic models. In order to accurately model the risk of these transitioning storms on communities such as Perth, the wind field of these storms needs to be parameterised for inclusion in stochastic models. These models allow large numbers of storms to be quickly simulated for use in risk modelling applications. Some authors have attempted to develop parameterisations that captures the unique shape of the surface wind field, with some recent success (Loridan et al. 2015), however an implementation for the Australian region has not yet been developed.
\end{abstract}

Geoscience Australia currently undertakes tropical cyclone risk assessments using a parameterised, 2D stochastic model called the Tropical Cyclone Risk Model (TCRM). TCRM uses parameterised wind fields to allow quick generation of thousands of tropical cyclones in order to develop a probabilistic understanding of tropical cyclone risk for Australia. At present, this model is not capable of simulating tropical cyclones undergoing ETT as a parameterisation of the wind field of these storms around Australia is not available. This work aims to explore ETT around Australia using a 3D, dynamical numerical weather prediction model with the ultimate goal of developing a parameterised wind field, suitable for inclusion in TCRM. This would allow risk assessments for these storms to be undertaken, and improve our understanding of the potential impact of such an event on large urban areas, such as Geraldton or Perth.

A modified version of the Weather Research and Forecast (WRF) model (Hybrid WRF) was used to simulate a number of hybrid idealised tropical cyclones, and steer them to undergo ETT. Hybrid WRF was developed to facilitate control over the track and location of landfall of a tropical cyclone, by introducing a steering flow to the boundary conditions of the model run. This method was used to steer a number of idealised tropical cyclones from off the northwest coast of Western Australia, south towards Perth, with the intent to force them to undergo ETT. Surface wind fields and other environmental characteristics (minimum pressure, latitude, thermal wind components, geopotential thickness and others) were analysed to determine the phase of ETT. This case study is the first example of Hybrid WRF being used to examine ETT, and while the steering flow did move the tropical cyclones into the extratropics as intended, only one storm was observed to undergo ETT. Further development of the code for Hybrid WRF is underway, with enhancements to permit time-varying lateral boundary conditions highlighted as a means to improve the realism of these experiments.

Based on these simulated events, we intend to develop time-evolving, storm-centred wind fields, as well as statistics on cyclone phase space parameters that can be used to determine the stage of transition to be used in a future stochastic-parametric model of tropical cyclones.

Keywords: Hazard, parametric model, risk, tropical cyclone 


\section{INTRODUCTION}

Geoscience Australia's Tropical Cyclone Risk Model (TCRM) is a parameterized, stochastic, cyclone risk model used to assess the wind hazard from tropical cyclones (TCs) along Australia's tropical coastline. The parameterized and simplified nature of the TCRM model allows the simulation of thousands of TCs, to develop a probabilistic understanding of cyclone risk around Australia. Tropical cyclones typically have an axisymmetric, warm-cored vortex that is readily represented by a simple radial profile, facilitating the use of the parameterised functions to simulate realistic cyclone profiles.

As TCs move out of the tropics, their fundamental characteristics change, and they become more extratropical in nature. Extratropical cyclones are driven by strong thermal gradients and as a result have a highly asymmetric wind field that is not captured by the TCRM TC wind field parameterisation. In order to simulate the risk of these transitioning TCs to Australia's mid-latitude coastal communities, a new parameterisation of the extratropical wind field needs to be developed, and incorporated into TCRM.

This study aims to test the workflow of a newly developed model framework designed to allow the steering of simulated TCs southwards to initiate ETT. The resulting wind field will be examined and used as the basis for developing an ETT wind field parameterization for future implementation in TCRM. This will be done by using historical ETT storms that affected Perth as a starting point for our analysis, initiating the experiments from the boundary conditions that led to their eventual transition.

\section{BACKGROUND}

Tropical cyclones can transition into extratropical storms as they move into the mid-latitudes if they encounter favourable conditions for re-intensification. Extratropical transition (ETT) events are most commonly seen in the western North Pacific, with $\sim 49 \%$ of all TCs in this region (over the period 19792004) transitioning into extratropical cyclones during their lifetime (Kitabatake 2011). Comparatively few TC events transition in the Indian Ocean ( 20\%; Foley et al. 1994) and South Pacific ( 33\%; Sinclair 2002) basins. The process of transitioning occurs when the storm begins to lose the characteristics of a tropical cyclone and becomes more extratropical in nature (Jones et al. 2003). This occurs as the storm interacts with mid-latitude environments, and is characterized by increased baroclinicity, increased wind shear, meridional humidity gradients, decreased sea surface temperature (or strong SST gradients) and an increased Coriolis force (Klein et al. 2000; Jones et al. 2003), which alter the structure and characteristics of the TC.

Hart (2003) developed an objective set of criteria for identifying ETT based on the concept of "phase space". The phase of a TC is determined by "classification of the three-dimensional nature of the cyclone structure", and considers changes in TC properties both at the surface, and in the upper troposphere. Hart's cyclone phase space identifies the structure of the cyclone based on three key parameters: thermal symmetry, thermal wind within the 900-600 hPa layer and thermal wind within the 600-300 hPa layer. TCs have a high degree of thermal symmetry, and a change to a thermally asymmetric structure can indicate interactions with baroclinic, mid-latitude environments. The vertical structure of a cyclone can be categorised as warm core or cold core depending on whether the isobaric height gradient of the cyclone centre increases (cold core) or decreases (warm core) with height. The differentiation between cold and warm core cyclones provides an indirect indicator of the "tilt" of the storm, indicating the degree of interaction with the baroclinic environment in the upper troposphere. Combination of Hart's three parameters allows for the objective categorisation of cyclone structure and transition.

\section{1. $\quad$ ETT in Australia}

Tropical cyclones are relatively common off Australia's west coast, with an average of seven cyclones forming in this region each season and $2-3$ of these crossing the WA coastline (Foley et al. 1994). As these systems move south into the mid-latitudes, most will weaken, however as many as $20 \%$ will intensify, forming potentially damaging extratropical cyclones (Foley et al. 1994). A climatology of wind speed across the southern Indian Ocean identified a minimum in wind speeds between $100-110^{\circ} \mathrm{E}$, just off the west coast of Western Australia, suggesting that systems that pass through this area are likely to intensify prior to moving east over Australia (Buckley et al. 2004). While the cyclone season for Western Australia is November to April, the majority of transitioning storms in the Southeast Indian Ocean occur in March and April (Jones et al. 2003). 


\subsection{Wind hazard risk for Perth}

The west coast of Western Australia is at risk from damage related to transitioning cyclones. From 1910 to 2004, a total of 14 tropical cyclones caused gales or wind-related damage to Perth, equating to an annual frequency of occurrence of 0.15 (storms that resulted in flooding but not wind damage were not included) (Jones et al. 2005). Wind hazard risk for Perth is greatest in early autumn, with over $70 \%$ of cyclones that have affected Perth occurring during March and April (Jones et al. 2005). A total of 13 cyclone events affecting Perth have been identified over the period 1843-2005, however the real number is likely to be larger, particularly for the pre-satellite era (Jones et al. 2005).

\section{Cyclone Alby, 1978}

The most damaging transitioning storm in Western Australia was Cyclone Alby, which impacted Perth in April 1978, costing an estimated \$39 million in damage, and the loss of five lives (Bureau of Meteorology 2016a). On the $2^{\text {nd }}$ April, Alby was at approximately $20^{\circ} \mathrm{S}$ and recurved towards the south-southeast, accelerating to an average speed of $25 \mathrm{~km} / \mathrm{h}$. Alby then started interacting with a cold front that caused it to transition into an extratropical storm as it moved further south (Jones et al. 2005). By the $2^{\text {nd }}$ April, Alby had begun to lose its symmetry, with the eye wall beginning to degrade and large cloud-free areas emerging to the east of the storm. By the $4^{\text {th }}$ April, Alby had reached translation speeds of $90 \mathrm{~km} / \mathrm{h}$, captured by the strong upper-level winds associated with the cold front (Bureau of Meteorology 1978; Jones et al. 2005). At this stage, the eye of the storm had completely degraded, and the storm consisted of a broad mass of cloud to the south of the eye (Jones et al. 2005).

Damage from Cyclone Alby was largely associated with wind rather than rain, with only light rainfalls recorded (mostly $<20 \mathrm{~mm}$ ) during the passage of Alby and its associated cold front (Bureau of Meteorology 1978). Mean wind speeds exceeding gale force $(63 \mathrm{~km} / \mathrm{h})$ were seen along the southwest coast of Western Australia, almost parallel to Alby's track (Bureau of Meteorology 1978). Wind gusts of up to $150 \mathrm{~km} / \mathrm{h}$ were seen throughout this region, with the strongest gust of $150 \mathrm{~km} / \mathrm{h}$ recorded at Albany on the $4^{\text {th }}$ April. Damage associated with these severe winds resulted in the destruction of forest plantation, crops, native forest, as well as widespread damage to roof cladding (Bureau of Meteorology 1978). Fires lit in agricultural and forest areas were fanned by the strong winds, burning over 110,000 ha of area and destroying farm infrastructure and equipment as well as causing extensive loss of livestock.

\section{Cyclone Ned, 1989}

Cyclone Ned formed as a low pressure system to the west of Broome on the $25^{\text {th }}$ March 1989 and lasted eight days before devolving into a weak low off southern Western Australia (Figure 1). It reached its maximum intensity as a category four cyclone on the $29^{\text {th }}$ March, with a central pressure of $941 \mathrm{hPa}$, and maximum 10 minute wind speeds of $166 \mathrm{~km} / \mathrm{h}$ (Bureau of Meteorology 2016b). Ned began to transition into an extratropical storm on the $30^{\text {th }}$ March under increasing vertical wind shear. A strong cold front approaching Perth on the $31^{\text {st }}$ March steered Ned back towards the Western Australia coast. Ned crossed the coastline near Perth at 2300 UTC on the $31^{\text {st }}$ March, bringing wind gusts of up to $110 \mathrm{~km} / \mathrm{h}$. Strong winds associated with $\mathrm{Ned}$ caused power disruptions and isolated roof damage (Bureau of Meteorology 2016b).

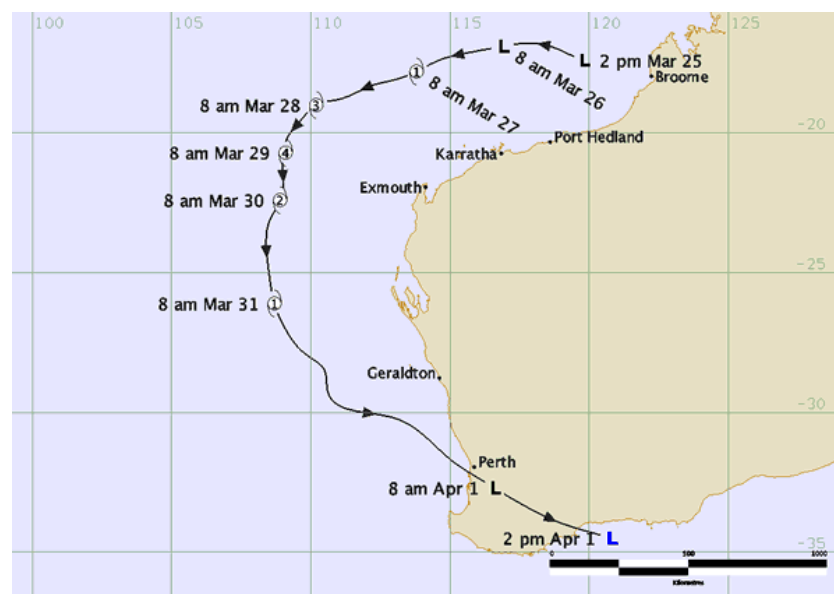

Figure 1. Track and intensity of Tropical Cyclone Ned (Bureau of Meteorology 2016b). 


\section{MODEL AND METHODS}

\subsection{Weather Research and Forecasting model (WRF)}

WRF is a numerical weather prediction model used for research and operational applications (Skamarock et al. 2008). The WRF source code is configurable for a range of applications, including real-time weather forecasting, data assimilation studies, parameterised physics research, regional and global climate simulations, air quality studies, atmosphere-ocean coupling and idealised simulations (Skamarock et al. 2008).

WRF can be run in two main modes

1. Ideal case; where the model domain is forced by prescribed conditions in an idealised domain.

2. Real case; where real-world meteorological observations, topography and conditions drive the model.

The real case can also be configured to run in real-time as a forecast model.

\subsection{Hybrid WRF}

The Hybrid WRF Cyclone Model (HWCM) was developed by Cindy Bruyere at UCAR and uses a combination of ideal and real WRF experiments to develop and steer a cyclone within WRF (Bruyere et al. 2016). A cyclone is first developed within an idealised WRF experiment, before being moved to a real WRF run that provides real-world context to the storm (Figure 2).

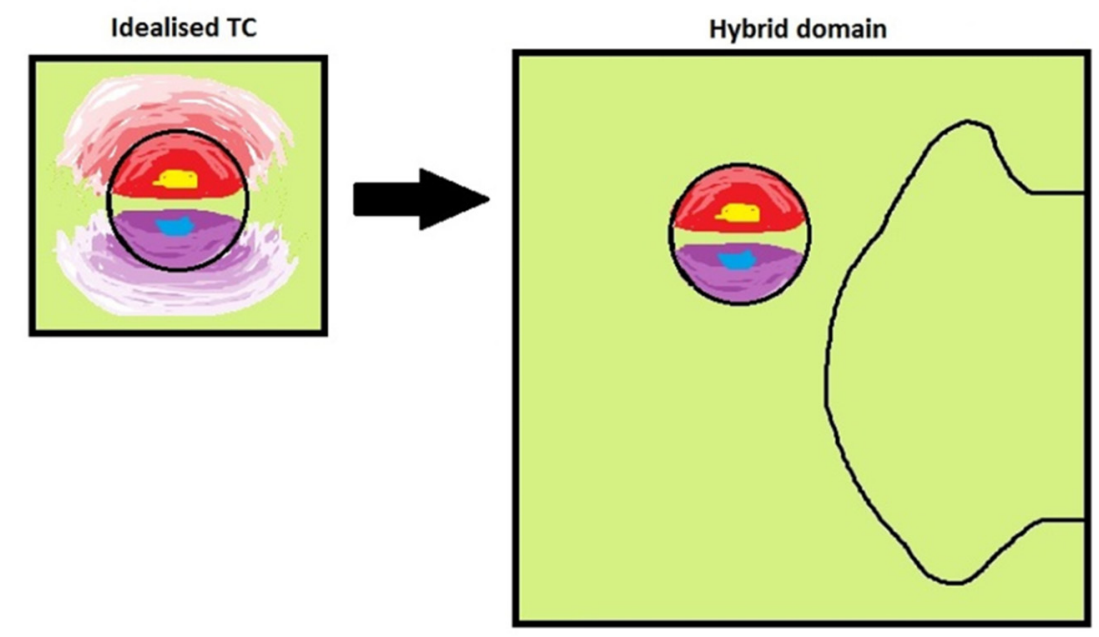

Figure 2. An idealized tropical cyclone is spun up in an idealized environment using ideal.exe before being placed within a hybrid domain. In this example, the tropical cyclone winds are placed into a domain with a constant southerly wind forcing, to steer the TC southwards.

Broadly, the HWCM involves three steps:

1. Set up and run an idealised tropical cyclone using the WRF ideal mode

2. Cut and paste the generated idealised cyclone into real-world boundary conditions, with the wind field modified to steer the TC (resulting in a combination of fields that are not in equilibrium with each other)

3. Run the hybrid WRF scenario using the WRF real mode

\subsection{Experimental design}

This study uses an experimental model workflow (HWRF) to attempt to model a transitioning tropical cyclone moving south off the Western Australian coastline. The cyclone is broadly designed to follow the historical paths of TC Alby (1978) and TC Ned (1989), both of which completed ETT and caused damage to Perth. Both of these systems were captured by a cold front as they moved south, assisting in their transition, and promoting their reintensification as an extra tropical storm. Reanalysis weather fields for these storms were used to recreate the conditions leading to ETT in an attempt to cause a model TC to undergo transition.

The HWCM was used to create an idealized tropical cyclone, which was then placed into reanalysis data fields from the time of TC Ned. The idealized storm was initialized using the idealized tropical cyclone 
configuration of WRF, which uses a default storm configuration described by Rotunno et al. (1987). Reanalysis data was examined from both TC Alby and TC Ned; however the experiments used reanalysis fields from ERA-interim of TC Ned - called the "hybrid-Ned" experiments from March and April 1989 as boundary conditions for the experiments (Figure 3). The HWRF workflow keeps the boundary conditions constant throughout the experiment, meaning a single ERA-interim time slice is used as the boundary conditions for the duration of the experiment. Initial run conditions were a combination of the ERA-interim timeslice, the synthetic tropical cyclone, and a southerly steering flow. Experiment Aus1 was run for 20 model days, but the remaining experiments were run for 12 model days, as this was shown to be a suitable length of time for the storm to move past Perth.

\section{RESULTS}

Simulations Aus1, Aus2 and Aus4 all failed to undergo ETT, with the storm instead weakening and moving into the Indian Ocean. In all three simulations, a high pressure cell developed to the south of the Australian mainland, overprinting the cold front in the run boundary conditions, and forcing the storm away from the coast.

Experiment Aus5 used boundary conditions prior to the arrival of a cold front to the west, and with the storm vortex placed further south, both in an attempt to force the front and the cyclone to interact during the experiment. A high pressure system developed to the south of Australia as in the other experiments, and the cyclone moved westward once it reached $\sim 30^{\circ} \mathrm{S}$, moving away from Perth and the Australian coastline (Figure 4).

Despite the inability to force the vortex and cold front to interact as the vortex moved southwards, this storm did begin to show signs of undergoing ETT, changing from a warm core to a cold core vortex by day seven (Figure 4). This was not accompanied by a change in vortex asymmetry, with the vortex remaining largely symmetrical as it weakened, indicating this storm did not complete the process of ETT.

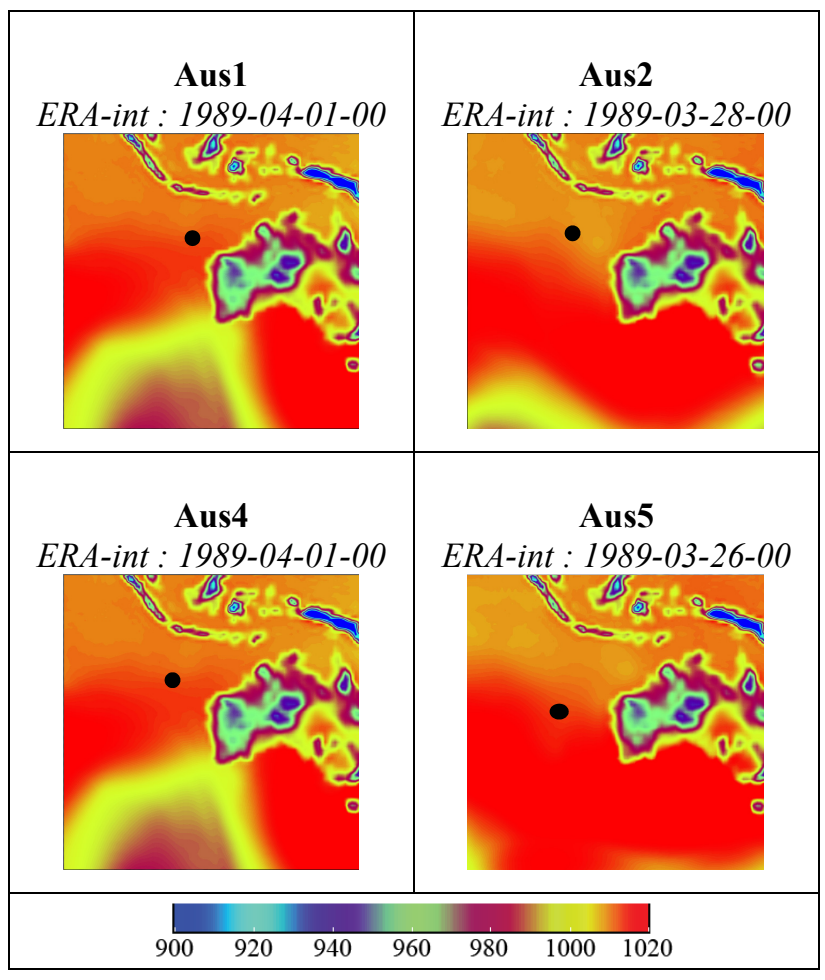

Figure 3. Initial surface pressure boundary conditions for the four hybrid Ned HWCM runs. MSLP boundary conditions $(\mathrm{hPa})$ show the differences in the relative development of the cold front between runs. The black dots represent the initial TC locations.

\section{DISCUSSION}

This project was a feasibility study intended to examine the nature of transitioning tropical cyclones within WRF with a view to developing a parameterisation scheme for ETTs for implementation in GA's tropical cyclone risk model. This study was able to demonstrate the potential of the HWCM for studies of ETT, with steering winds applied to the model runs successfully steering the model tropical cyclone southwards as intended.

The importance of the initial and lateral boundary conditions in facilitating ETT was demonstrated by the development of a high pressure system to the south of Australia in all four experiments. This system prevented the storms from moving south past Perth and instead steered them westward, where they dissipated without undergoing ETT. The variations in initial and boundary conditions across experiments did not seem to have any impact on the development of this high pressure system; even in experiments with a strong cold front to the south of Perth, a high pressure system quickly developed. This was caused by the current method for creating the model initial conditions, which combine reanalysis conditions with a synthetic TC, and apply a uniform steering flow, which involves replacing equilibrium reanalysis boundary conditions across the model domain with a uniform wind field. Once the experiment was initiated, the model needed to reequilibrate, resulting in the development of the climatological high pressure system to the south of Australia. This occurred at the expense of the boundary conditions, which were forced from the edge of the model domain, and were washed out by the model equilibration. This could be addressed in future model runs by 
applying the steering flow on top of reanalysis conditions, reducing the size of the perturbation to equilibrium conditions, and forcing the model with time varying boundary conditions, which may increase the influence of features such as cold fronts by better simulating their evolution through time.

The GFDL vortex tracker is a post processing tool available from UCAR, which identifies and tracks a cyclone vortex throughout a model run (or forecast), producing diagnostics relating to cyclone nature, structure and phase (Bao et al. 2012). The vortex tracker uses GRIB input, produced by the Unified Post Processor, run following the production of WRF output files. These tools were explored for this study but have not yet been integrated into this workflow. Without this quantitative analysis, qualitative analysis of cyclone phase space was undertaken using geopotential height anomalies and geopotential height perturbations, following figure three of Hart (2003; Figure 4). These diagrammatic phase space indicators provided a useful analysis of cyclone phase, and were able to record a change in phase in experiment Aus5, but could be improved in the future.
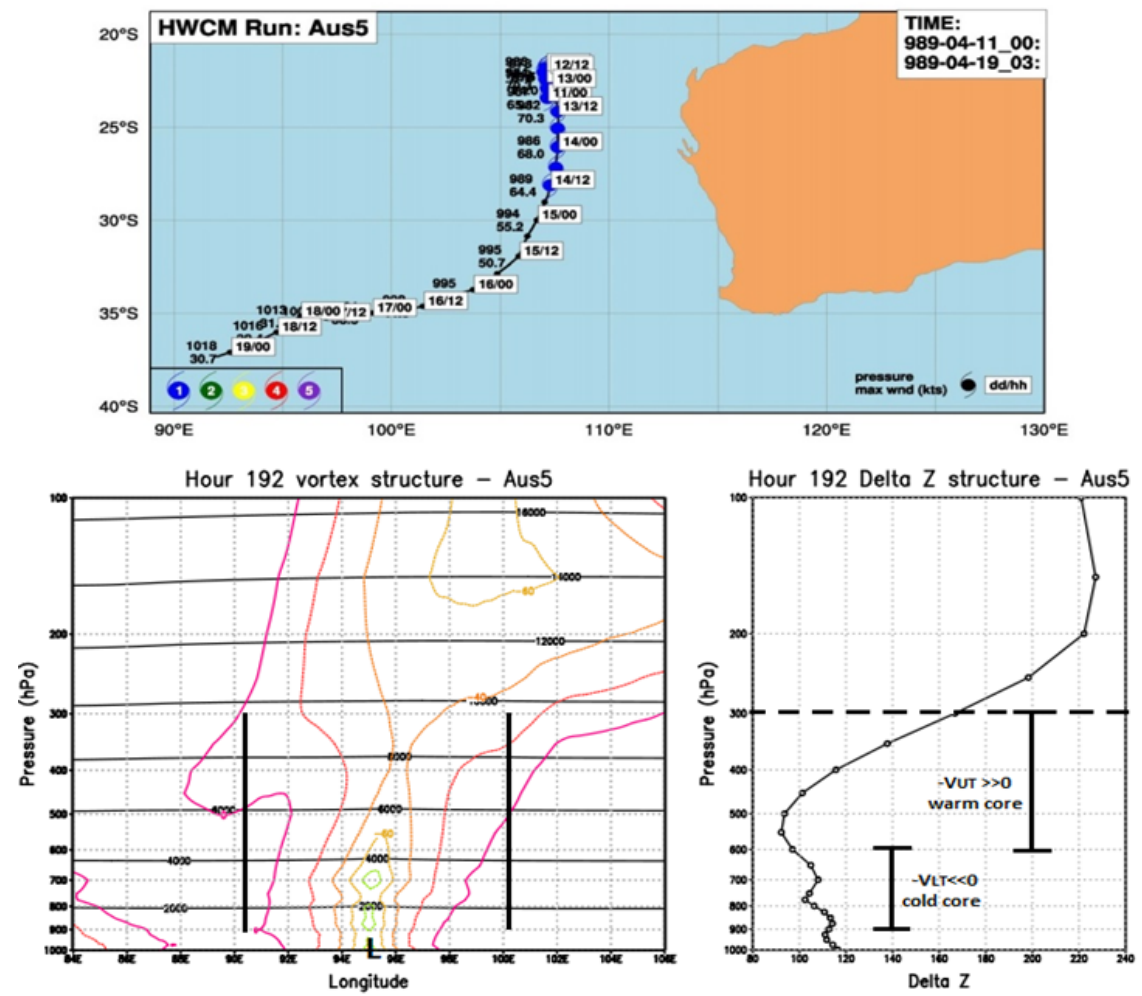

Figure 4. Aus5 cyclone track (top), and qualitative analysis of cyclone phase for Aus5, showing a cold core, symmetric vortex at hour 192. Bottom left: Longitudinal cross section through the centre of the vortex showing geopotential height (solid black horizontal lines) and geopotential height anomalies (coloured contours). The two vertical lines indicate the $500 \mathrm{~km}$ cyclone radius. Bottom right: Height difference within this radius for each pressure level. Bottom figures follow the method of Hart (2003) for identifying ETT.

\section{CONCLUSIONS AND RECOMMENDATIONS}

This project demonstrated the feasibility of using the HWCM to force idealised tropical cyclones southward. While none of the experiments produced a cyclone which had undergone ETT, one experiment showed a change in core structure, suggesting that additional experiments could be expected to undergo ETT under the right conditions. This work is ongoing, and future work will continue to develop the capability of HWRF to model ETT, with a view to developing a parameterisation scheme for implementation into TCRM. The next steps for this work will include:

- Integration of a steering flow into reanalysis conditions. The replacement of equilibrium weather conditions with a uniform steering flow causes the model to be initiated out of equilibrium. If the steering flow can be applied on top of existing conditions, this would remove the need for re-equilibration, and allow the model to respond to designed changes in lateral boundary conditions, such as the passage of a cold front.

- Time-varying boundary conditions for forcing interaction of TC with low pressure fronts. The current HWCM workflow involves using a single reanalysis file to force the boundary conditions for the 
- duration of the model experiment. Future work should explore the use of time varying boundary conditions to force the movement of e.g. a cold front across the model domain. This would allow the model to be forced from the lateral boundaries with more appropriate reanalysis conditions and may help to develop and maintain a cold front within the model domain.

- Use of the Unified Post Processor and GFDL vortex tracker to analyse cyclone phase space. Further debugging of the Unified Post Processor and GFDL vortex tracker is required to integrate this tool into the HWRF workflow. Once these programs are available to use, they will provide valuable diagnostics of cyclone phase space through time. This would improve on the qualitative analysis used in this study to diagnose cyclone phase at designated time slices throughout the model run.

- Exploration of additional initial conditions for promoting ETT. In this project, we only used reanalysis input from ERA-interim from one period in time - the $26^{\text {th }}$ March to $10^{\text {th }}$ April 1989 - which coincided with the timing of Cyclone Ned. Additional time periods e.g. around the time of Cyclone Alby, could also be used as initial conditions. Further experimentation of initial boundary conditions may reveal a more suitable meteorological configuration for promoting ETT.

\section{ACKNOWLEDGEMENTS}

This paper is published with the permission of the CEO, Geoscience Australia

\section{REFERENCES}

Bao, S., D. Stark and L. Bernardet (2012). Users' guide for the community release of the GFDL Vortex Tracker, The Developmental Testbed Center.

Bruyere, C. L., G. J. Holland, B. Buckley, M. Leplastrier, P. Chan, M. Tingley and A. Dyer (2016). Hybrid Real-Idealized Tropical Cyclones as a New Loss Tool. AMOS National Conference. Melbourne, Australia, February 2016.

Buckley, B. W. and L. M. Leslie (2004). Preliminary climatology and improved modelling of South Indian Ocean and Southern Ocean mid-latitude cyclones. International Journal of Climatology 24: 1211 1230 .

Bureau of Meteorology (1978). Tropical Cyclone Alby 27/03/1978 to 04/04/1978. in Tropical Cyclone Reports: 9.

Bureau of Meteorology. (2016a). "Tropical Cyclone Alby." Retrieved 20/06/16, from http://www.bom.gov.au/cyclone/history/wa/alby.shtml.

Bureau of Meteorology (2016b). Tropical Cyclone Ned. Perth Tropical Cyclone Warning Centre.

Evans, J. L. and R. E. Hart (2003). Objective Indicators of the Life Cycle Evolution of Extratropical Transition for Atlantic Tropical Cyclones. Monthly Weather Review 131(5): 909-925.

Foley, G. R. and B. N. Hanstrum (1994). The Capture of Tropical Cyclones by Cold Fronts off the West Coast of Australia. Weather and Forecasting 9(4): 577-592.

Hart, R. E. (2003). A cyclone phase space derived from thermal wind and thermal asymmetry. Monthly Weather Review 131: 585 - 616.

Jones, S. C., P. A. Harr, J. Abraham, L. F. Bosart, P. J. Bowyer, J. L. Evans, D. E. Hanley, B. N. Hanstrum, R. E. Hart, F. Lalaurette, M. R. Sinclair, R. K. Smith and C. Thorncroft (2003). The Extratropical Transition of Tropical Cyclones: Forecast Challenges, Current Understanding, and Future Directions. Weather and Forecasting 18(6): 1052-1092.

Jones, T., M. Middelmann and N. Corby (2005). Natural hazard risk in Perth, Western Australia, Geoscience Australia: pp 352.

Kitabatake, N. (2011). Climatology of Extratropical Transition of Tropical Cyclones in the Western North Pacific Defined by Using Cyclone Phase Space. Journal of the Meteorological Society of Japan. Ser. II 89(4): 309-325.

Klein, P. M., P. A. Harr and R. L. Elsberry (2000). Extratropical Transition of Western North Pacific Tropical Cyclones: An Overview and Conceptual Model of the Transformation Stage. Weather and Forecasting 15(4): 373-395.

Loridan, T., S. Khare, E. Scherer, M. Dixon and E. Bellone (2015). Parametric modeling of transitioning cyclone wind fields for risk assessment studies in the Western North Pacific. Journal of Applied Meteorology and Climatology 54: $624-642$.

Rotunno, R. and K. A. Emanuel (1987). An air-sea interaction theory for tropical cyclones. Part II: Evolutionary study using a nonhydrostatic axisymmetric numerical model. Journal of the Atmospheric Sciences 44(3): 542-561.

Skamarock, W. C., J. B. Klemp, J. Dudhia, D. O. Gill, D. M. Barker, M. G. Duda, X.-Y. Huang, W. Wang and J. G. Powers (2008). A description of the Advanced Research WRF Version 3. NCAR Technical Note, NCAR: $113 \mathrm{pp}$. 